Rev Inv Vet Perú 2014; 25(2): 333-339

doi: http://doi.org/10.15381/rivep.v25i2.8507

\title{
Comunicación
}

\section{CARACTERIZACIÓN DE UN BROTE DE LA ENFERMEDAD DE GUMBORO EN UNA GRANJA AVÍCOLA DE LA HABANA}

\author{
Characterization of a Gumboro Disease Outbreak in a Poultry \\ FARM OF HaVANa
}

\author{
Raiden Grandía G. ${ }^{1,3}$, Abdulahi Alfonso M. ${ }^{2}$, Bárbara O. González N. ${ }^{1}$
}

\section{Resumen}

El objetivo del estudio fue caracterizar un brote de la enfermedad de Gumboro en una granja avícola de La Habana, Cuba, en 2010. Se estudió una población de 2959 pollos de engorde, analizándose los antecedentes de crianza, observaciones clínicas y hallazgos anatomopatológicos. Se calcularon los índices de morbilidad, mortalidad y letalidad. Se realizó la necropsia a 40 aves con signos clínicos para el estudio histopatológico de la bolsa de Fabricio, timo y bazo y se determinó la presencia de antígenos virales en macerado bursal mediante un AC-ELISA. Se evidenció una mortalidad de 5.1\%, morbilidad de $8.4 \%$ y letalidad de $60.6 \%$. El signo clínico más frecuente fue la depresión general (100\%) y las lesiones más frecuentes fueron bursitis hemorrágica, edema bursal y nefritis (8590\%), habiendo depleción linfoide folicular en los cortes histológicos de bolsa. El 82.5\% de las bolsas de Fabricio fueron positivas en el AC-ELISA. Las deficiencias en la vacunación y bioseguridad condicionaron el establecimiento del brote, causando pérdidas económicas en la granja.

Palabras clave: caracterización, brote, Gumboro, pollo de engorde, La Habana

\section{Abstract}

The objective of the study was to characterize a Gumboro disease outbreak in a poultry farm of Havana, Cuba in 2010. A population of 2959 broilers was studied. Breeding management, clinical observations and anatomopathological findings were analyzed. The morbidity, mortality and lethality rates were calculated. Necropsy was done in 40

\footnotetext{
${ }^{1}$ Centro Nacional para la Producción de Animales de Laboratorio, CENPALAB, La Habana, Cuba

${ }^{2}$ Centro Nacional de Sanidad Agropecuaria, CENSA, Mayabeque, Cuba

${ }^{3}$ E-mail: raiden@cenpalab.inf.cu
}

Recibido: 24 de octubre de 2013

Aceptado para publicación: 3 de febrero de 2014 
birds with signs of illness for the histopathological study of the bursa of Fabricius, thymus and spleen. The presence of virus antigens in bursal macerated was conducted by an AC-ELISA. The study showed 5.1\% mortality, $8.4 \%$ morbidity, and $60.6 \%$ lethality. The most frequent clinical sign was general depression (100\%) and macroscopic lesions were hemorrhagic bursitis, edema of the bursa and nephritis (85-90\%). Follicular lymphoid depletion in bursa was found in the histopathology. The AC-ELISA showed that $82.5 \%$ of bursas were positive. Vaccination and biosecurity deficiencies conditioned the occurrence of the Gumboro disease outbreak, causing economic losses in the farm.

Key words: characterization, outbreak, Gumboro, broiler, Havana

\section{INTRODUCCIÓN}

El sector avícola mundial ha tenido un notable crecimiento en las últimas décadas; sin embargo, la presencia de enfermedades en los centros de producción ha seguido causando grandes pérdidas económicas. Asimismo, las aves son muy sensibles al estrés ambiental, por lo que se deben extremar los esfuerzos para mantener a las aves en ambientes de confort y con las debidas medidas de bioseguridad (Nillipour, 2000).

Todo tipo de estrés puede afectar el sistema inmune de las aves (Wright, 1995). Valladares (2010) hace referencia a varios factores inmunosupresores, incluyendo enfermedades como la anemia infecciosa, Marek, leucosis aviar, reticuloendoteliosis y Gumboro.

La enfermedad de Gumboro afecta la bolsa de Fabricio, especialmente en aves jóvenes. Es causada por un Avibirnavirus de la familia Birnaviridae, con un genoma compuesto por un ARN de doble cadena (Jackwood, 2009), estructurado por un segmento A que codifica para las proteínas virales VP2, VP3, VP4 y VP5, y por el segmento B que codifica para la proteína VP1 (Jackwood, 2009; Pan et al., 2009).

La distribución global de esta enfermedad está condicionada por las vías de transmisión utilizadas por el virus (oral y conjuntival), por la alta resistencia que tiene frente a tratamientos térmicos $\left(56{ }^{\circ} \mathrm{C}\right.$ por $\left.5 \mathrm{~h}\right)$ y químicos (éter, cloroformo) (Avellaneda y
Villegas, 1995), y por la supervivencia en materiales de crianza (encamado, agua y alimentos) por largos periodos de tiempo (50125 días) (Fernández et al., 1997; Alfonso y Noda, 2012). Además, el virus también puede infectar aves silvestres (Egaña, 2010).

En Cuba, la enfermedad de Gumboro se reportó por primera vez en 1982 (Venereo et al., 1982). Fue controlada luego de algunos años con la vacuna de tipo intermedia Nobilis D-78, hasta que en 1992 ocurrió un incremento de la incidencia, condicionada por cambios de la cepa vacunal y en el esquema de vacunación utilizado, unido a una disminución en las medidas de bioseguridad debido a las condiciones impuestas por un periodo económicamente difícil para el país («periodo especial»). Desde entonces, los brotes de la enfermedad han aumentado, reportándose una mortalidad superior a los 50 mil pollos de engorde por año (Alfonso y Noda, 2012).

La enfermedad afecta poblaciones de aves en el continente americano (Stoute et al., 2009; Villacrés et al., 2010), donde Cuba no está exenta (Alfonso y Noda, 2012). La elevada morbilidad (100\%), letalidad (superior al 50\%) y considerable mortalidad (550\%) (Avellaneda y Villegas, 1995; Egaña, 2010; Alfonso y Noda, 2012), ocasionan grandes pérdidas económicas a la industria avícola, dependiendo en gran medida de la virulencia de la cepa presente (Rauf et al., 2011). Este estudio tuvo como objetivo caracterizar un brote de la enfermedad de Gumboro presentado en una granja avícola de La Habana. 


\section{Materiales y Métodos}

El estudio se realizó en una masa clínicamente afectada $(n=249)$ por un brote sospechoso de la enfermedad de Gumboro en una población de 2959 pollos de engorde, de ambos sexos, a partir de los 19 días de edad.

Se seleccionaron 40 animales de 24 días de edad con signos de enfermedad o moribundos. La necropsia de las aves se realizó en el Laboratorio de Patología del Centro Nacional para la Producción de Animales de Laboratorio (CENPALAB). Se tomaron muestras de bolsas de Fabricio, timos y bazos $(n=20)$, fijadas en formalina tamponada al $10 \%$, incluidas en parafina, cortadas a $4 \mu \mathrm{m}$ y teñidas con Hematoxilina-Eosina para el análisis histopatológico.

Asimismo, se separaron porciones de las 40 bolsas de Fabricio y se llevaron al Laboratorio de Virología Aviar del Centro Nacional de Sanidad Agropecuaria (CENSA) para la confirmación de la presencia de antígenos virales del virus de la enfermedad de Gumboro en macerados bursales, mediante un AC-ELISA. Se consideraron como muestras positivas aquellas con una densidad óptica (DO) de dos o más desviaciones estándares (DE) y como negativas aquellas con una DE o menos en relación con la DO del control positivo, considerándose como dudosos los valores intermedios. Se utilizó un lector de ELISA (VersaMaxPLUS ROM v. 1.21) a una longitud de onda de $450 \mathrm{~nm}$.

En el diagnóstico presuntivo se analizaron los antecedentes de la crianza, las observaciones clínicas y los hallazgos morfopatológicos en animales afectados.

Se recopiló información sobre mortalidad y sacrificio, aves enfermas y lesiones anatomopatológicas (macroscópicas) para calcular los índices de morbilidad, mortalidad y letalidad. Además, se calculó el consumo de alimento, peso a los 42 días de edad y conversión alimenticia. Se realizaron distribuciones de frecuencias absolutas y relativas con el programa InfoStat v. 2.0. Asimismo, se realizó la prueba de comparación de proporciones individuales $(\mathrm{p}<0.05)$, tanto para los signos clínicos como las lesiones macroscópicas, aplicando las pruebas de Fisher $(\mathrm{F})$ y Duncan con el programa COMPAPRO v. 2.1.

En la valoración económica se consideraron las pérdidas en pesos cubanos (CUP) y en dólares americanos (USD) por concepto de disminución de ingresos relacionados con la mortalidad, sacrificio sanitario, decomiso de canales afectadas e incremento de gastos relacionados con el consumo de alimento y medicinas.

\section{Resultados y Discusión}

La viabilidad en el lote muestreado fue de $92.3 \%$, el consumo de alimento de 3850 g/ave, el peso a los 42 días de edad de $1.75 \mathrm{~kg}$ y la conversión alimenticia de 2.2, indicadores que pueden ser considerados aceptables a pesar de la presentación de la enfermedad de Gumboro. Estos resultados son muy similares a otros estudios en condiciones adecuadas de salud (Ayala et al., 2006; Fumero et al., 2007).

La administración oportuna de antibióticos para prevenir infecciones secundarias, y del complejo vitamínico B como reconstituyente estimulante del sistema inmunológico (Alahyari-Shahrasb et al., 2012), así como la inclusión de medidas de bioseguridad inmediatas dirigidas a la contención del brote, permitió una rápida recuperación de la masa animal y, por lo tanto, sin afectar en exceso los índices productivos y económicos. Avellaneda y Villegas (1995) señalan que las consecuencias económicas de la enfermedad de Gumboro están condicionadas en gran medida por los decomisos de canales, el uso de antibióticos y la mortalidad. 
Cuadro 1. Frecuencia de presentación de signos clínicos en 249 pollos de engorde afectados por la enfermedad de Gumboro

\begin{tabular}{lc}
\hline Signos clínicos & $\begin{array}{c}\text { Frecuencia } \\
(\%)\end{array}$ \\
\hline Picaje (cloaca) & $24.8^{\mathrm{a}}$ \\
Diarreas blancas & $44.9^{\mathrm{b}}$ \\
Plumas erizadas & $59.0^{\mathrm{c}}$ \\
Postración & $69.8^{\mathrm{d}}$ \\
Depresión general & $100.0^{\mathrm{e}}$ \\
\hline
\end{tabular}

a,b,c,d,e Superíndices diferentes indican diferencias estadísticas entre signos clínicos $(p<0.0001 ; F=81.69)$

En Cuba el programa establecido para el control de esta enfermedad por inmunización es mediante la vacunación asistida por serología a fin de inmunizar la población antes que los anticuerpos maternos desciendan a niveles no protectivos y que el virus vacunal colonice la bolsa de Fabricio antes que el virus de campo (Perera et al., 2005); sin embargo, no se hizo esta evaluación a las aves que ingresaron a la granja. Además, tampoco fueron vacunadas las reproductoras antes de la puesta, lo cual pudo ser un factor favorable adicional para el establecimiento del brote (Avellaneda y Villegas, 1995; Perera et $a l ., 2005)$. Este incumplimiento rompió con el esquema cubano recomendado para lograr una inmunización eficaz contra esta enfermedad.

La habilitación sanitaria se realizó adecuadamente antes de la recepción de las aves, aunque el vaciado sanitario fue solo de cuatro días, cuando lo establecido es un mínimo de 10 días para reducir considerablemente la carga viral ambiental (Grezzi, 2001). Por otro lado, se pudieron identificar varias brechas sanitarias como el no mantenimiento del cordón sanitario, filtro sanitario ineficiente, lavado y desinfección insuficiente de manos, vestuario y calzado, disposición de cadáveres tardía, no periodicidad en la desratización y desinsectación, calidad microbiológica del agua no aceptable y un inadecuado sistema de inmunización. Estos fallos facilitaron el establecimiento y desarrollo del brote en la población susceptible (Nillipour, 2000).

En relación a los signos clínicos, el total de aves afectadas manifestó depresión general (Cuadro 1). Los signos clínicos observados son compatibles con la enfermedad de Gumboro (Avellaneda y Villegas, 1995; Valladares, 2010), aunque no se observaron temblores e incoordinación (Insua et al., 2005). Asimismo, la morbilidad del brote fue relativamente baja $(8.4 \%)$.

La mortalidad causada por la enfermedad de Gumboro ( $\mathrm{n}=151)$ fue de $5.1 \%$; no obstante se registraron 192 muertes y 40 sacrificios sanitarios indicando una mortalidad de $7.7 \%$ en toda la crianza. El $5.1 \%$ de mortalidad se encuentra dentro del rango de 5 a $50 \%$ señalado por Avellaneda y Villegas (1995).

La letalidad del virus en las aves enfermas fue elevada (60.6\%), resultado que coincide con otros estudios en Cuba donde demuestran índices superiores al 50\% (Alfonso y Noda, 2012). Por otra parte, este hallazgo indica la presencia de cepas muy virulentas, pues más del $90 \%$ de las cepas caracterizadas en el país corresponden a este subtipo (Alfonso et al., 2013).

La ocurrencia de manifestaciones clínicas y de muertes comenzó a los 22 días de edad; es decir, al tercer día del traslado de las aves a la nave de crecimiento, coincidiendo con el periodo de incubación de 2-3 días, de acuerdo a Avellaneda y Villegas (1995), aunque el Terrestrial Animal Health Code de la OIE (2013) señala siete días como periodo de incubación. Es posible que el virus haya estado latente en la nave de crecimiento o que las aves hayan estado infectadas antes del traspaso y el estrés haya sido un factor condicionante para el desarrollo de la enfermedad (Wright, 1995). 
Cuadro 2. Frecuencia de presentación de lesiones macroscópicas en 40 pollos de engorde afectados por la enfermedad de Gumboro

\begin{tabular}{lc}
\hline Lesiones macroscópicas & $\begin{array}{c}\text { Frecuencia } \\
(\%)\end{array}$ \\
\hline Proventriculitis & $7.5^{\mathrm{a}}$ \\
Atrofia bursal & $10.0^{\mathrm{a}}$ \\
Hemorragias en músculo & $47.5^{\mathrm{b}}$ \\
Nefritis & $85.0^{\mathrm{c}}$ \\
Edema bursal & $85.0^{\mathrm{c}}$ \\
Bursitis hemorrágica & $90.0^{\mathrm{c}}$ \\
\hline a,b,c Superíndices diferentes indican \\
diferencias estadísticas entre lesiones \\
(p<0.0001; F=23.71)
\end{tabular}

La bursitis hemorrágica difusa fue la lesión macroscópica observada con mayor frecuencia en las 40 aves necropsiadas, seguida del edema bursal y la nefritis (Cuadro 2). Las lesiones encontradas forman parte del cuadro descrito para la enfermedad de Gumboro (Avellaneda y Villegas, 1995; Fernández et al., 1997; Jordan y Pattison, 1998; Valladares, 2010). Sin embargo, no se observó bursitis caseosa-hemorrágica, que fuera descrita por Fernández et al. (1997) en la enfermedad de Gumboro.

En el estudio histopatológico se observaron cambios circulatorios en el $80 \%$ de las bolsas de Fabricio, como congestión, edema, hemorragias e infiltración de macrófagos,
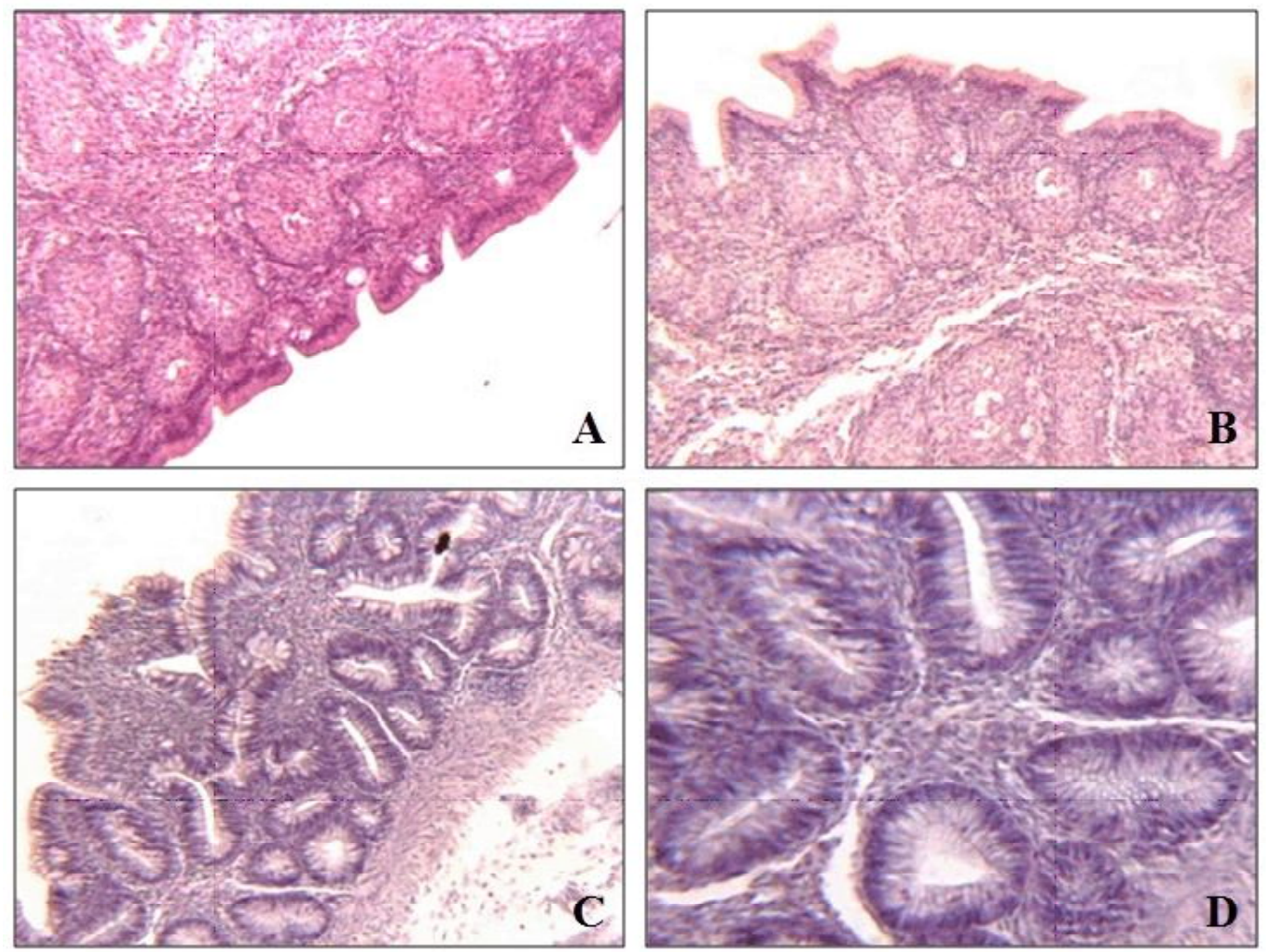

Figura 1. Cortes histológicos de la bolsa de Fabricio. A: Congestión, microhemorragias y edema en el estroma interfolicular. Los folículos corticales y medulares con espacios vacíos por la despoblación linfoide y en la parte superior izquierda necrosis y abundantes detritos celulares. H-E (100x). B: Congestión y edema con depleción linfoide e infiltrado de macrófagos. H-E (100x). C: Atrofia folicular por pérdida de células linfoides produciéndose una invaginación del epitelio, adoptando la forma de glándulas mucosas. H-E (100x). D: Vista de C en mayor aumento (200x) 
además de depleción linfoide de moderada a severa con atrofia folicular, en cerca de la mitad de los folículos (Fig. 1A,B). Estos cambios coinciden con reportes de la literatura (Calnek, 2000). Según Babaahmady et al. (2005), las lesiones agudas de la bolsa se presentan a los 3-4 días de la infección, fundamentalmente, la necrosis linfoide, además de hiperemia y edema.

La degeneración y necrosis linfoide, así como la apoptosis, se hallaron tanto en la región cortical como medular de la bolsa. En el $20 \%$ de los casos se observó el pliegue bursal con intensa atrofia y marcado plegamiento folicular, acompañados de degeneración quística epitelial e intensa depleción linfoide (Fig. 1C,D), tal y como lo describen Babaahmady et al. (2005) y Valladares (2010), quienes señalaron que la atrofia folicular es consecuencia de la intensa depleción de linfocitos debida a la necrosis y apoptosis celular. Por otro lado, los cortes de timo y bazo no evidenciaron lesiones degenerativas ni necrosis en el tejido linfoide.

Se detectaron antígenos del virus de la enfermedad de Gumboro en 33 de las 40 muestras analizadas $(82.5 \%)$ por el ACELISA. Esta es una prueba de alta sensibilidad y especificidad que permite procesar un gran volumen de muestras, convirtiéndose en una herramienta eficaz para la confirmación del virus de campo en poblaciones afectadas (Cardoso et al., 1998; OIE, 2006).

En el análisis de la valoración económica, como consecuencia del brote, se dejó de ingresar \$2320 CUP por la pérdida de 232 animales y se gastaron $\$ 128.90$ USD en medicamentos.

\section{Literatura Citada}

1. Alahyari-Shahrasb M, Moravej H, Shivazad M. 2012. Efecto de diferentes niveles de premezcla vitamínica durante el período de finalización en el com- portamiento de pollos de ceba y la inmunocompetencia en sistemas de jaula de batería y en piso. Rev Cub Cienc Agr 46: 279-285.

2. Alfonso AM, Martínez OP, Dolz, R, Valle R, Perera CL, Bertran K, Frías MT, et al. 2013. Spatiotemporal phylogenetic analysis and molecular characterization of infectious bursal disease viruses based on the VP2 hyper-variable region. PLoS One 8(6): e65999.

3. Alfonso A, Noda J. 2012. Análisis retrospectivo del comportamiento epizootiológico de la enfermedad de Gumboro en Cuba. REDVET 13(10). [Internet], [4 febrero 2013]. Disponible en: http://www.veterinaria.org/revistas/ redvet/n101012.html

4. Avellaneda G, Villegas P. 1995. Enfermedad infecciosa de la bolsa (Gumboro). Avic Prof 12: 190-200.

5. Ayala L, Martínez M, Acosta A, Oraida D, Hernández L. 2006. Una nota acerca del efecto del orégano como aditivo en el comportamiento productivo de pollos de ceba. Rev Cub Cienc Agr 40:455-458.

6. Babaahmady E, Joa R, Noda J. 2005. Enfermedad de Gumboro. Histopatología de la Bursa de Fabricio en la enfermedad natural y experimental en pollos de engorde. REDVET 6(3). [Internet], [10 octubre 2012]. Disponible en: http:// www.veterinaria.org/revistas/redvet/ n040405.html

7. Calnek BW. 2000. Enfermedades de las aves. $2^{\mathrm{a}}$ ed. México DF: El Manual Moderno. $1110 \mathrm{p}$.

8. Cardoso TC, Sousa RLM, Alessis AC, Montassier HJ, Pinto AA. 1998. A double antibody sandwich ELISA for rapid diagnosis of virus infection and to measure the humoral response against infectious bursal disease on clinical material. Avian Pathol 27: 450-454.

9. Egaña SCL. 2010. Estudio serológico de exposición a agentes infecciosos en fardela negra (Puffinus griseus, Gmelin 1789) provenientes de una colonia reproductiva en Isla Guafo, Chile. Tesis 
de Médico Veterinario. Valdivia, Chile: Universidad Austral de Chile. 44 p.

10. Fernández R, Montiel E, Jaramillo C, Ramos Y, Jackwood D, Quesada J, Matzer, N. 1997. Caracterización de las cepas del virus de la enfermedad de Gumboro en aves de engorde en Latinoamérica, utilizando la técnica de reacción en cadena de la polimerasa. En: XV Congreso Latinoamericano de Avicultura. Cancún, México. p 119-122.

11. Fumero JE, Godínez O, Torres R. 2007. Comportamiento productivo de un híbrido cubano para la producción de carne. Rev Cub Cienc Avícola 31(1): 57-62.

12. Grezzi G. 2001. Bioseguridad en la industria avícola: mitos y realidades. Avic Prof 19(2): 12-32.

13. Insua RG Silveira EAP, Olazábal EM. 2005. Frecuencia y caracterización de lesiones anatomo-patológicas en la enfermedad de gumboro y enfermedades secundarias asociadas en nuestras condiciones ambientales. Estudio retrospectivo. REDVET 6(10). [Internet], [10 octubre 2012]. Disponible en: http:// www.veterinaria.org/revistas/redvet/ n101005.html

14. Jackwood DJ. 2009. Current status of infectious bursal disease. Food Animal Health Research Program. Ohio, USA. En: XXXIV Convención Anual ANECA. Acapulco, México.

15. Jordan FTW, Pattison M. 1998. Enfermedades de las aves. $3^{\mathrm{a}}$ ed. México DF: El Manual Moderno. 522 p.

16. Nillipour AH. 2000. Tropical wall and how to minimize a detrimental effects. World Poultry 12: 41-42.

17. [OIE] World Organisation for Animal Health. 2006. Standard Operating Procedure (SOP) for OIE Validation and Certification of Diagnostic Assays. Version 1.9.

18. [OIE] World Organisation for Animal Health. 2013. Terrestrial Code. Infectious bursal disease (Gumboro disease). Chapter: 10.8.1. [Internet], [29 diciembre 2013]. Disponible en: http:// www.oie.int/en/international-standard- setting/terrestrial-code/access-online/ ?htmfile=chapitre_1.10.8.htm

19. Pan J, Lin L, Tao YJ. 2009. Selfguanylylation of birnavirus VP1 does not require an intact polymerase activity site. Virology 395: 87-96.

20. Perera CL, Noda J, Cuello S, Alfonso P, Espinosa V, Merino A. 2005. Vacunación asistida por serología para la enfermedad infecciosa de la bolsa. REDVET 6(5). [Internet], [1 octubre 2012]. Disponible en: http://www.veterinaria.org/revistas/redvet/n050505.html

21. Rauf A, Khatri M, Murgia MV, Jung K, Saif YM. 2011. Differential modulation of cytokine, chemokine and Toll like receptor expression in chickens infected with classical and variant infectious bursal disease virus. Vet Res 42: 85. doi:10.1186/1297-9716-42-85

22. Stoute ST, Jackwood DJ, SommerWagner SE, Cooper GL, Anderson ML, Woolcock PR, Bickford AA, et al. 2009. The diagnosis of very virulent infectious bursal disease in California pullets. Avian Dis 53: 321-326.

23. Valladares JC. 2010. Experiencias prácticas en el diagnóstico de la infección de la Bolsa de Fabricio. [Internet], [10 octubre 2012]. Disponible en: http:// www.engormix.com/MA-avicultura/sanidad/articulos/experiencias-practicasdiagnostico-infeccion-t2904/165-p0.htm

24. Venereo M, Fonseca C, Espinosa V. 1982. Primer reporte de la bursitis infecciosa aviar en Cuba. Rev Cub Cienc Vet 13: 29-42.

25. Villacrés $C$, Rodríguez-Hidalgo $R$, Torres ML, Urresta D, Benítez-Ortiz W. 2010. Caracterización molecular del virus de la enfermedad de Gumboro a través de la transcripción en reversa-reacción en cadena de la polimerasa combinado con análisis de enzimas de restricción. REDVET 11(9). [Internet], [10 octubre 2012]. Disponible en: http:// www.veterinaria.org/revistas/redvet/ n090910.html

26. Wright C. 1995. La cumbre de Gumboro. Industria Avícola 42(7): 12-22. 